\title{
Penser la musique, entendre la société. Un parcours à l'EHESS
}

\section{Marta Amico}

\section{(2) OpenEdition}

1 Journals

Édition électronique

URL : http://journals.openedition.org/transposition/1755

DOI : 10.4000/transposition. 1755

ISSN : 2110-6134

Éditeur

CRAL - Centre de recherche sur les arts et le langage

Référence électronique

Marta Amico, «Penser la musique, entendre la société. Un parcours à l'EHESS », Transposition [En ligne], Hors-série 1 | 2018, mis en ligne le 30 janvier 2018, consulté le 10 février 2020. URL : http:// journals.openedition.org/transposition/1755; DOI : 10.4000/transposition.1755

Ce document a été généré automatiquement le 10 février 2020.

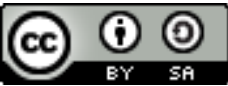

La revue Transposition est mise à disposition selon les termes de la Licence Creative Commons Attribution - Partage dans les Mêmes Conditions 4.0 International. 


\title{
Penser la musique, entendre la société. Un parcours à l'EHESS
}

\author{
Marta Amico
}

Depuis l'Italie, l'EHESS paraissait un autre monde. La figure de Jean-Loup Amselle revenait dans les cours de Licence en anthropologie que je fréquentais à l'université de Turin, ainsi que d'autres noms tels que celui de Marc Augé, Michel Agier ou Didier Fassin. Mais je n'aurai pas songé de franchir un jour le seuil de ce temple de la discipline, encore moins d'y faire un doctorat. Le hasard en décida autrement. Arrivée à Paris en 2006 pour un Erasmus en ethnomusicologie à la Sorbonne, sans connaitre un seul mot de français, je fus rapidement introduite à la culture de la résistance qui peut enflammer à tout moment les étudiants de la capitale. Alors que je m'apprêtais à fréquenter mes premiers cours au Boulevard Saint-Michel, les portails de la mythique université parisienne furent cadenassés, cachés derrière des murs de métal, présidés par des policiers en armes qui défendaient l'édifice des jeunes militants contre la loi CPE. Le projet de connaissance qui m'avait amenée à franchir les Alpes pour joindre cette capitale historique des savoirs était soudain en péril. Il n'y avait qu'une seule voie de sauvetage. Traverser le Jardin du Luxembourg et m'aventurer dans les méandres du $6^{\mathrm{e}}$ arrondissement jusqu'au Boulevard Raspail, dans l'une des rares institutions parisiennes à donner encore des cours. Avant que ce lieu ne tombe aussi, par un coup porté par des vandales me dit-on, je fis la rencontre d'autres étudiants comme moi, Italiens, Brésiliens, Espagnols, Congolais, Vietnamiens, tous engagés dans le monde vivace, multilingue, critique et dense d'attentes du Master 1 en Anthropologie sociale et Ethnologie.

2 La décision de joindre officiellement "l'École », comme on l'appelait affectueusement entre étudiants, vint lorsque, de retour à Turin, un professeur se montra perplexe face à mon envie d'étudier la World Music à partir d'un groupe de musiciens touaregs qui jouaient de la guitare électrique. Selon lui, il fallait aller d'abord dans les campements nomades, en plein désert, étudier les traditions anciennes et les instruments « de làbas ». Puis j'aurais pu, plus tard, regarder cette « contamination », comme il l'appelait. Cette image de l'altérité musicale comme pureté et de la rencontre comme 
contamination, convoquée par mon professeur, ne me convainquait pas. Je pensais alors la chose dans ces termes : "Certes une distance tangible me sépare des musiciens du désert, mais cet ailleurs n'est-il pas aussi une dimension incontournable de mon monde musical?» Ce questionnement fut accueilli avec enthousiasme par Denis Laborde, qui tenait à ce moment un séminaire sur la World Music à l'EHESS et accepta de diriger ma thèse. Il fut nourri des conseils d'Esteban Buch, qui m'aguilla sur les études au sujet de la musique dans des contextes de violence, de Michael Werner, qui anima un séminaire sur la notion féconde d'« histoire croisée » et d'Emmanuel Olivier, qui me rassura sur le fait que l'on peut encore faire de l'ethnomusicologie en Afrique sans jeter un regard compatissant sur les effets de la mondialisation. Ces quatre perspectives méritaient chacune qu'on s'y attarde un peu, pour mieux comprendre ce qu'était cette science sociale qu'on appelait «musique» dans ce lieu qui ne ressemblait à aucun autre.

3 Venant du système universitaire italien, la structuration des cours à l'EHESS m'était totalement nouvelle. Pas de contrôle des connaissances, pas d'obligation de fréquence, pas d'hégémonie de l'examen, pas d'impression d'être une étudiante, mais de faire partie d'un groupe, un comité, un cénacle de chercheurs qui débattaient de ce qui les emportaient, apprenant à d'autres à faire de même. Lors des séminaires, des invités se succédaient chaque semaine, offrant des perspectives inattendues, parfois encore trop difficiles à cerner pour une chercheuse en herbe comme moi. Mais les questionnements qui se soulevaient là me tenaient accrochée. L'EHESS devenait ma maison, celle qui conjuguait l'approche musicale aux sciences sociales, l'engagement dans les grands débats théoriques à l'art de la description ethnographique, la pratique solitaire de l'enquête aux projets collectifs.

4 Au fil des séminaires, je commençais à regarder mon objet de recherche autrement et à forger une nouvelle pensée. Un numéro de L'Homme venait de sortir, intitulé « Musique et anthropologie». Il affirmait que les ethnomusicologues ont un "devoir de mémoire » envers des "esthétiques perdues ", et qu'il fallait s'occuper en priorité des musiques « historiquement plus enracinées [...] en apprenant à les comprendre dans leur contexte de production ${ }^{1}$ ». J'essayais de mieux comprendre cet objectif, affirmé dans une revue prestigieuse. La musique touarègue serait-elle une "esthétique perdue »? Pas vraiment, si l'on considère que l'un des groupes phares du nord du Mali était alors affiché massivement dans les couloirs du métro parisien à l'occasion d'un concert dans la prestigieuse salle de l'olympia. Encore moins si l'on écoute une musicienne, qui me dit que la «musique traditionnelle touarègue » avait été créée en 1995 dans un camp de réfugiés en Mauritanie pour obtempérer la demande d'un festival en Belgique. J'avais alors des doutes sur l'ancrage culturel et territorial de la "musique touarègue », qui mettaient en question les délimitations géographiques de mon terrain.

5 Ceci m'approcha des études critiques de l'ethnomusicologie notamment anglo-saxonne et américaine, qui postulent une discipline « autoréflexive, 'rapatriée' et nourrie par le discours postcolonial ${ }^{2} »$. Est-il possible d'étudier la musique touarègue autrement que par le filtre d'une " esthétique perdue », et donc insoluble avec les normes de "notre » musique ? Et, si oui, comment prendre en compte les efforts collectifs qui construisent une altérité musicale et la célèbrent ensuite comme objet premier de la World Music? Ceci pose évidemment des questions épistémologiques. Quel rôle a pu jouer le discours ethnomusicologique dans la réification et la légitimation scientifique d'un imaginaire 
collectif de l'altérité musicale ? Et que peut-on dire si on déplace les mobiles de l'Autre et qu'on reconnait avec Ramon Pelinski le «je en tant qu'altérité et sa propre identité en tant que différence ${ }^{3}$ "? Denis Laborde me rassura en invitant dans l'un de ses séminaires Philip Bohlman, qui nous présenta la World Music en affirmant que «les ethnomusicologues ne peuvent pas simplement observer et décrire l'espace entre le soi et l'autre, ils doivent plutôt y rentrer et gérer les formes complexes que prend la rencontre dans cet espace. Cet espace s'ouvre comme l'endroit où se produit le terrain ${ }^{4}$ ».

6 Creuser cet espace défie la pertinence des aires culturelles. La présence importante de spécialistes de l'Afrique à l'EHESS m'aida à chercher les branchements ${ }^{5}$ entre les répertoires musicaux touaregs et les dynamiques historiques et politiques qui caractérisaient le Mali et le Sahara au début des années 2000. Pourtant, si de par mon terrain j'étais classée d'emblée au rang des "africanistes », la façon de décliner l'objet me plaçait aussi dans une optique plus large, qui questionnait les partages disciplinaires et la constitution historique du regard anthropologique sur l'Afrique par le filtre de la musique. Ce questionnement était d'actualité au sein de l'École, comme en témoigne le sociologue Michel Wieviorka, pour qui la "notion d"“aires culturelles" implique de découper le monde à l'aide de catégories qui sont non pas ou pas nécessairement celles des populations ou groupes concernés, mais celles de ceux qui catégorisent, et dont les représentations sont elles-mêmes alors à analyser ${ }^{6}{ }$. Mais alors peut-on étudier les Touaregs en ethnomusicologue non-africaniste? Peut-on écrire une "histoire croisée» de la musique ${ }^{7}$ à partir des représentations postcoloniales de l'Afrique qui habitent l'Europe d'aujourd'hui ?

7 Ceci amène à des questions de méthode. Où se placer pour observer des musiciens, des concerts, des festivals, comment décrire les passerelles entre le désert du Sahara et la World Music sans entrer dans le piège des jugements esthétiques et des discours paternalistes sur les «traditions menacées" qui ont traversé les premières considérations ethnomusicologiques sur la mondialisation culturelle ? Pour contourner ces écueils, je parcourais cette distance entre une "posture d'observateur", qui renvoie aux outils dont on se dote pour conduire nos observations, et un "poste d'observation ", la place que nous occupons dans les situations que nous décrivons ${ }^{8}$. Ceci amène à interroger la place que nous occupons dans la fabrication des musiques que nous nous chargeons d'observer. Selon l'anthropologue et philosophe Gérard Lenclud :

les données servant à constituer les faits portent en elles les marques indélébiles de l'activité conceptuelle de l'observateur, lequel serait bien en peine d'être ce voyageur « purement empirique » dont Emmanuel Kant se moqua9.

8 L'aveu de cette co-construction de l'objet ouvre de nouvelles pistes d'analyse. Plutôt que partir des théories générales sur le rapport entre la musique et la mondialisation, ou la tradition ou encore la performance ou le concert, comme des données brutes de l'expérience qui offriraient un modèle préalable à appliquer aux faits observés, j'interrogeais des itinéraires de vie, des formes musicales, des répertoires, des discours, des dispositifs de mise en scène, des technologies d'enregistrement, des lieux de musique. Cette approche promeut une anthropologie pragmatique de la musique qui place l'observation de la pratique au premier rang des conduites d'analyse. Cela implique de s'intéresser, dans le sillage de Denis Laborde, à ce qui fait être la musique : 
qu'est-ce qui fait être, ici ou là, des pratiques musiciennes que les syntagmes de World Music ou «musiques du monde » permettent de désigner ${ }^{10}$ ?

Des projets vinrent s'ancrer sur ces questionnements, fédérant des groupes de doctorants autour de l'institution "voisine » de l'EHESS sur le Boulevard Raspail, la Maison des Cultures du Monde, puis au Festival créole de Berlin, puis encore à la Fondation Royaumont, au Festival Villes des Musiques du Monde en banlieue parisienne et à la Philharmonie de Paris. Ce fut pendant ces périodes d'« ethnographie collective ", comme on aimait l'appeler avec d'autres doctorants de Denis Laborde, que j'appris à me défaire des visions communes de la musique comme un objet strictement artistique pour laisser la place à un questionnement plus large sur les processus d'institution de l'objet «musique » dans nos sociétés, reliant le geste d'un créateur, le moment d'émotion collective du concert, les compétences techniques déployées lors d'un sound-check et les politiques publiques de la « démocratisation culturelle ». Ainsi la musique n'était plus une essence, un ensemble d'œuvres ou un fait donné que je pouvais isoler et regarder au microscope, mais le fruit d'une manière de faire, que j'apprenais à considérer dans sa complexité.

10 La fin de mon doctorat marqua la période des questionnements sur les voies professionnelles possibles pour une docteure en sciences sociales. Je découvris à ce moment avec une certaine surprise que les engagements dans le monde de la culture par lesquels j'avais appris à faire l'anthropologie pouvaient se transformer en un travail rémunéré et reconnu par les institutions. Il y avait une demande, de la part des acteurs culturels, une remise en question, un tâtonnement face à des commandes politiques ou des objectifs sociaux à accomplir, et les chercheurs étaient appelés à participer à la réflexion. Ce regard sur et dans les instituions m'aida à mieux cerner les outils de ma pensée, qui étaient devenus presque naturels pour moi, à en penser le rôle social, la pertinence dans le "faire", dans l'engagement civil. Des collaborations avec les doctorants et chercheurs, des participations aux séminaires, des multiples projets me tenaient ancrée à l'EHESS alors même que je commençais à voler avec mes propres ailes. Je réfléchissais alors au statut paradoxal de la musique : la place qu'elle occupe dans nos sociétés est inversement proportionnelle à celle qui lui est destinée à l'université, celle d'une discipline marginale, ancillaire par rapport à d'autres thèmes forts des sciences sociales. Mais alors, comment donner de l'épaisseur à cet objet multiforme, comment perfectionner des outils pour l'analyser du point de vue de ses contenus aussi bien que des processus de production de ces contenus? Comment décrire l'institution de cette catégorie «musique» sans perdre de vue les manifestations qui nous provoquent tant d'émotions? Comment produire un mode de connaissance qui traverse les barrières disciplinaires et provoque une dynamique d'interaction entre les chercheurs et avec d'autres acteurs engagés dans le monde musical contemporain?

11 Aujourd'hui, fraichement élue Maitresse de conférence en Ethnomusicologie, le moment est venu d'agir de l'intérieur sur le paysage universitaire, et de transmettre à d'autres le langage appris pendant mon parcours à l'EHESS. Ainsi j'ose espérer que le regard qui s'est forgé Boulevard Raspail irrigue le champ des études sur la musique à d'autres endroits, fabriquant le futur d'une vision commune ou d'une "école de pensée ». 


\section{BIBLIOGRAPHIE}

AMSELLE, Jean-Loup, Branchements. Anthropologie de l'universalité des cultures, Paris, Flammarion, 2001.

BOHLMAN, Philip, World Music. A Very Short Introduction, Oxford, Oxford University Press, 2002.

EL GHADBAN, Yara, « Chants et contrechamps de l'ethnomusicologie. Essai bibliographique », Anthropologie et société 30 (2006), p. 219-235.

FAVRET SAADA, Jeanne, Les Mots, la mort, les sorts : la sorcellerie dans le bocage, Paris, Gallimard, 1977.

LABORDE, Denis, Les musiques à l'école, Paris, Bertrand Lacoste, 1998.

LENCLOUD, Gérard, « Grand résumé de 'L'Universalisme ou le pari de la raison. Anthropologie, histoire, psychologie' ", Sociologies 2013, en ligne : http://journals.openedition.org/sociologies/ 4614 (consulté le 18 janvier 2018).

LOTART-JACOB, Bernard et OLSEN, Myriam, « Musique, anthropologie. La conjonction nécessaire ", L'Homme 171-172 (2004), p. 7-26.

PELINSKI, Ramon, «L'ethnomusicologie à l'ère postmoderne », Musiques : une encyclopédie pour le XXI ${ }^{e}$ siècle, vol. II, Arles, Actes Sud, 2004, p. 740-765.

WERNER, Michael et ZIMMERMAN, Bénédicte, De la comparaison à l'histoire croisée, Paris, Seuil, 2004.

WIEVIORKA, Michel, « Faut-il encore parler d'aires culturelles? », intervention au colloque «Aires culturelles », Paris, 5 novembre 2014, publié dans le carnet de recherche Michel Wieviorka, sociologue, en ligne : http://wieviorka.hypotheses.org/347 (consulté le 9 janvier 2018).

\section{NOTES}

1. LOTART-JACOB, Bernard et OLSEN, Myriam, «Musique, anthropologie. La conjonction nécessaire ", L'Homme 171-172 (2004), p. 23.

2. EL GHADBAN, Yara, "Chants et contrechamps de l'ethnomusicologie. Essai bibliographique », Anthropologie et société 30 (2006), p. 224.

3. PELINSKI, Ramon, «L'ethnomusicologie à l'ère postmoderne », Musiques : une encyclopédie pour le XXI siècle, vol. II, Arles, Actes sud, 2004, p. 750.

4. BOHLMAN, Philip, World Music. A Very Short Introduction, Oxford, Oxford University Press, 2002, p. 37. Traduction de l'anglais de l'auteur.

5. AMSELLE, Jean-Loup, Branchements. Anthropologie de l'universalité des cultures, Paris, Flammarion, 2001.

6. WIEVIORKA, Michel, «Faut-il encore parler d'aires culturelles?», intervention au colloque «Aires culturelles », Paris, 5 novembre 2014, publié dans le carnet de recherche Michel Wieviorka, sociologue, en ligne : http://wieviorka.hypotheses.org/347 (consulté le 9 janvier 2018).

7. WERNER, Michael et ZIMMERMAN, Bénédicte, De la comparaison à l'histoire croisée, Paris, Seuil, 2004.

8. FAVRET SAADA, Jeanne, Les Mots, la mort, les sorts : la sorcellerie dans le bocage, Paris, Gallimard, 1977. 
9. LENCLOUD, Gérard, « Grand résumé de 'L'Universalisme ou le pari de la raison. Anthropologie, histoire, psychologie' », Sociologies (2013).

10. LABORDE, Denis, Les musiques à l'école, Paris, Bertrand Lacoste, 1998.

\section{AUTEUR}

\section{MARTA AMICO}

Marta AMICO est Maitresse de Conférences en ethnomusicologie à l'Université de Rennes 2. Après un doctorat à l'EHESS de Paris, elle a été chercheuse au King's College de Londres (Angleterre) avec une bourse Marie Curie de l'Union européenne, au Musée du Quai Branly, à la Fondation Maison des Sciences de l'Homme de Paris, à la Philharmonie de Paris et au Center for World Music de l'Université de Hildesheim (Allemagne).

Ses travaux récents étudient les processus de création et de patrimonialisation musicales qui se composent dans des contextes de conflit armé, notamment au Mali et au Sahara. Plus largement, elle s'intéresse aux relations entre musique, identité, globalisation, politiques culturelles et maintien de la paix. Un autre filon de recherche interroge les pratiques collectives de la musique qui se développent en France au sein de projets musicaux dits « à vocation sociale ». 